\title{
Multi-scale mechanics of traumatic brain injury: predicting axonal strains from head loads
}

\author{
R. J. H. Cloots - J. A. W. van Dommelen - S. Kleiven • \\ M. G. D. Geers
}

Received: 24 September 2011 / Accepted: 28 February 2012 / Published online: 21 March 2012

(C) The Author(s) 2012. This article is published with open access at Springerlink.com

\begin{abstract}
The length scales involved in the development of diffuse axonal injury typically range from the head level (i.e., mechanical loading) to the cellular level. The parts of the brain that are vulnerable to this type of injury are mainly the brainstem and the corpus callosum, which are regions with highly anisotropically oriented axons. Within these parts, discrete axonal injuries occur mainly where the axons have to deviate from their main course due to the presence of an inclusion. The aim of this study is to predict axonal strains as a result of a mechanical load at the macroscopic head level. For this, a multi-scale finite element approach is adopted, in which a macro-level head model and a micro-level critical volume element are coupled. The results show that the axonal strains cannot be trivially correlated to the tissue strain without taking into account the axonal orientations, which indicates that the heterogeneities at the cellular level play an important role in brain injury and reliable predictions thereof. In addition to the multi-scale approach, it is shown that a novel anisotropic equivalent strain measure can be used to assess these micro-scale effects from head-level simulations only.
\end{abstract}

Keywords Traumatic brain injury - TBI - Diffuse axonal injury · DAI · Injury criteria $\cdot$ Head model $\cdot$ Finite element method $\cdot$ Multi-scale

R. J. H. Cloots · J. A. W. van Dommelen $(\bowtie) \cdot$ M. G. D. Geers

Materials Technology Institute, Eindhoven University

of Technology, P.O. Box 513, 5600 MB Eindhoven,

The Netherlands

e-mail: j.a.w.v.dommelen@tue.nl

S. Kleiven

Division of Neuronic Engineering, School of Technology and Health, Royal Institute of Technology, 14152 Huddinge, Sweden

\section{Introduction}

The brain is a vulnerable part of the human body in case of an accident, such as in a road traffic crash situation. Based on 23 reports in different European countries, 15 per 100,000 people die because of traumatic brain injury (TBI) each year (Tagliaferri et al. 2006). Diffuse axonal injury (DAI) is one of the most frequently occurring types of TBI (Gennarelli et al. 1982). It is primarily involved with dynamic noncontact loading, although it is believed to occur in closed head impacts as well (Gentleman et al. 1995; Smith et al. 2003).

In order to improve the prevention and diagnosis of TBI, a better understanding of the relation between mechanical loading and TBI is necessary. Therefore, brain injury criteria are developed that can predict TBI as the result of a mechanical load. The most used brain injury criterion in the automotive industry nowadays is the head injury criterion (HIC), which is based on head-level kinematics (NHTSA 1972). Although the mechanical loading occurs at the head level, injury of the brain is often the result of more local mechanical phenomena. Because of this, more sophisticated brain injury criteria are needed to recover the local mechanics. For this purpose, three-dimensional finite element (FE) head models are developed that simulate the response to a mechanical loading of the head to assess the risk of TBI (e.g., Takhounts et al. 2003; Zhang et al. 2004; Kleiven 2007; Marjoux et al. 2008). FE head models have a good potential to predict DAI, since they describe local deformations within the brain (Miller et al. 1998; Smith et al. 2003). However, an indisputably well-defined correlation between mechanical loading and DAI using FE head models has not been achieved yet. A possible explanation is that in most of the currently used head models, anisotropic mechanical behavior of brain tissue is not included, even though experimental studies have 
concluded that neural tissue behaves clearly anisotropically in some regions of the brain (e.g., Arbogast and Margulies 1998, 1999; Prange and Margulies 2002; Nicolle et al. 2005; Ning et al. 2006; Hrapko et al. 2008). In line with this, recent studies have been performed that take the axonal orientation into account leading to tissue strains in the axonal direction (Chatelin et al. 2011; Wright and Ramesh 2012). Nevertheless, even if tissue strains could be predicted accurately by head models, the link to real injury is still not straightforward, as several studies concerning TBI have shown that tissue strains lead to injury at a cellular level (e.g., Bain et al. 2001; Engel et al. 2005; Floyd et al. 2005; Morrison III et al. 2006; Cater et al. 2006; Elkin et al. 2007), whereby the microstructural heterogeneities at the cellular level are of influence.

Brain tissue contains neurons and glial cells, which both consist of a cell body from which processes (i.e., axons and dendrites) extend (Marieb 1998; Nolte 2002). The diameters of the cell bodies are about $5 \mu \mathrm{m}$ for the glial cells and often less than $10 \mu \mathrm{m}$ for nonpyramidal cells and up to $20 \mu \mathrm{m}$ for other cortical and hippocampal neurons (Rajkowska and Goldman-Rakic 1995; Rajkowska et al. 1998; Pierri et al. 2001; Cotter et al. 2002; Highley et al. 2003; Hutsler 2003). The cell bodies are mainly residing in the cortex, but their axons, which have a uniform diameter, extend into the white matter regions and can be many centimeters long (Alberts et al. 1994).

Axonal injury typically occurs where the direction of axons changes or at the location of a change in tissue-density (e.g., gray-white matter interface near the cerebral cortex) (Gaetz 2004). The first type of injury occurs for example in the brainstem and the corpus callosum and is described by Povlishock (1993), who observed that axonal injury was present at locations where the axons have to deviate from a straight path because of an obstruction (e.g., a blood vessel or a cell body). In a previous study (Cloots et al. 2011), mechanical heterogeneities at the axonal level, leading to axons being subjected to locally higher strains than the tissue-level strains, were investigated as a possible source for this pathological observation. Based on a micro-level critical volume element (CVE), it was concluded in Cloots et al. (2011) that the geometrical configuration of deviating axons near an inclusion leads to increased axonal loading and that, therefore, if present, these regions may be critical for axonal injury.

This influence of the microstructure of the tissue might induce an orientation dependence of the sensitivity of brain tissue to a mechanical load in regions where the tissue has a unidirectionally oriented structure. This highlights the importance of taking into account microstructural aspects of the tissue in tissue-level brain injury criteria.

Within this context, the aim of this study is to investigate the local axonal strains near an inclusion in relation to the tissue-level strains of the brainstem and the corpus callosum during mechanical loading of the head. To achieve a coupling between an FE head model that does not contain details at a cellular-level and local axonal strains, a multi-scale framework with a macroscopic head model and a microscopic critical volume element (CVE) is used. Both levels are solved with the FE method. The CVE is constructed on the basis of microscopic pathological findings for DAI. An approximate single-scale approach to include cellular-level effects in the same FE head model (without using a CVE) is pursued through an anisotropic equivalent strain measure that can estimate axonal strains directly from head model tissue strains (Cloots et al. 2012). To investigate to capability of the anisotropic equivalent strain measure to account for microstructural effects (as modeled in a CVE), the outcome of both approaches will be compared.

\section{Methods}

In this section, first, the multi-scale method will be explained, whereas the anisotropic equivalent strain measure will be explained further on. For the multi-scale approach, at the macro level ${ }^{1}$, an FE head model is used and at the micro level a CVE is developed (see Fig. 1). To achieve this, the influence of the microstructural configuration as well as the anisotropic sensitivity of the tissue to mechanical loads should be accounted for. The FE head model is originally developed by Kleiven (2007), and for the current study, it is extended with anisotropic tissue behavior. The CVE developed in this study represents a microstructural configuration that is critical for axonal injury. In Fig. 2, it is shown schematically how the head model and the CVE are coupled in the multi-scale framework. Macroscopic loading conditions are based on the reconstruction of a real sports accident (Viano et al. 2005; Kleiven 2007) and are imposed on the head model. After the simulations of the head model are completed, the obtained internal brain tissue loading response from the head model is used as a loading condition for the CVE, where for each integration point in the regions of interest of the head model, a separate CVE simulation is conducted. The outcome of the CVE simulations is the axonal loading, where the orientation of the axons is taken into account. For completeness, a situation is also simulated in which no inclusion is present in the microstructure by directly taking the logarithmic tissue strain obtained in the head model in the macroscopic local main axonal direction (i.e., without a CVE simulation).

\footnotetext{
1 The macro-level model is concerned with a length scale of several decimeters, whereas the micro-level modeling approach is sizeindependent, as explained further on. Therefore, the terms macro and micro (including microstructure) should be interpreted in a relative manner, where the micro level is considered to be much smaller than the macro level.
} 


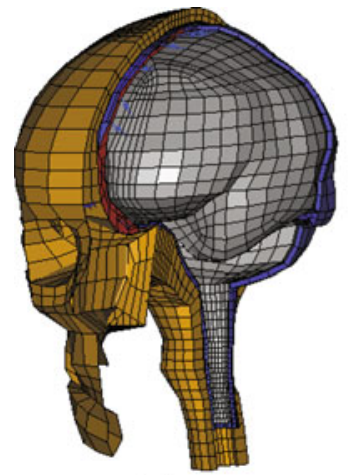

(a)

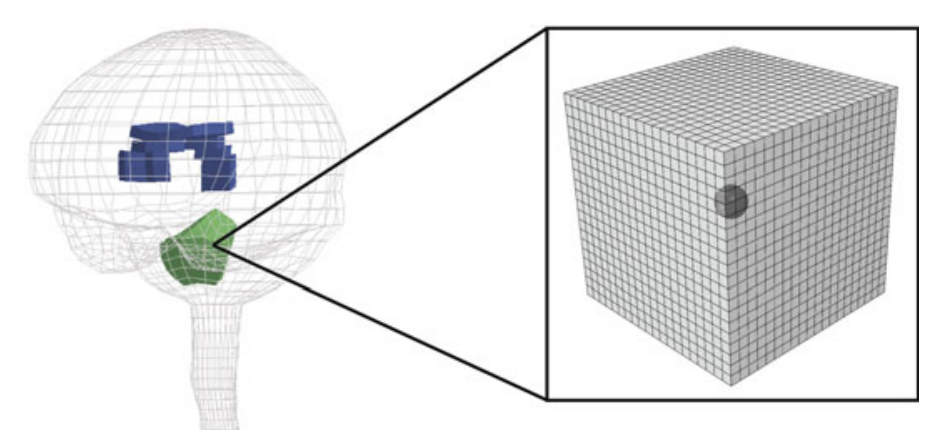

(c)

Fig. 1 Models at the macroscopic and the microscopic level: a head model, b head model showing the brainstem and the corpus callosum and c critical volume element

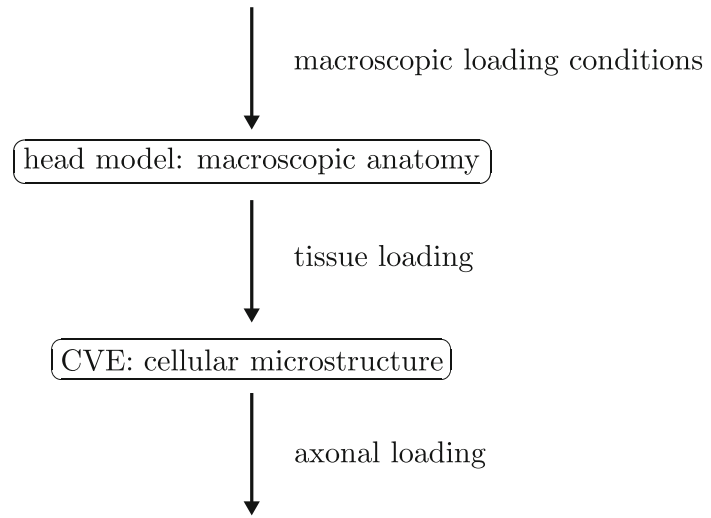

Fig. 2 Schematic representation of the computational multi-scale implementation

Since the discrete focal impairments of the axons are often found in the brainstem and the corpus callosum, focus is put on these parts of the head model. In order to perform an indepth study of the axonal strains in the CVE in relation to the tissue strains in the brainstem and the corpus callosum of the head model, one specific injurious loading case of the head model is considered, where for each element of the brainstem and the corpus callosum, a CVE simulation is performed.

\subsection{Head model}

The head model used in this study is based on the model developed by Kleiven (2007) in the FE code LS-DYNA 971 (LTSC, Livermore, CA) and contains the skull, the scalp, the brain, the meninges, the cerebrospinal fluid (CSF), and eleven pairs of the largest parasagittal bridging veins (see Fig. 1a). Furthermore, it includes the ventricles and it distinguishes between different parts within the brain, such as the corpus callosum and the brainstem (see Fig. 1b). The head model consists of 11,158 eight-node hexahedral elements, of which 7,128 represent the brain, 10,165 four-node shell and membrane elements, and 22 two-node truss elements. The simulations are performed in the dynamic regime with an explicit integration scheme. For this study, the material behavior of the brain tissue is described by a viscoelastic fiber-reinforced anisotropic material model. This model is implemented as a user material of which the hyperelastic strain energy potential is:

$$
\begin{aligned}
W= & \frac{G}{2}\left(\tilde{I}_{1}-3\right)+K\left(\frac{J^{2}-1}{4}-\frac{1}{2} \ln J\right) \\
& +\frac{k_{1}}{2 k_{2}}\left(e^{k_{2}\langle\tilde{E}\rangle^{2}}-1\right),
\end{aligned}
$$

where the third term on the right-hand side is based on the Holzapfel-Gasser-Ogden form (Gasser et al. 2006), with only one fiber family here, with

$\tilde{E}=\kappa\left(\tilde{I}_{1}-3\right)+(1-3 \kappa)\left(\tilde{I}_{4}-1\right)$,

where $G$ is the shear modulus, $K$ is the bulk modulus, $\tilde{I}_{1}$ is the first invariant of the isochoric right Cauchy-Green deformation tensor $\tilde{\boldsymbol{C}}=J^{-\frac{2}{3}} \boldsymbol{C}$ with $\boldsymbol{C}$ the right Cauchy-Green deformation tensor and $J=\operatorname{det}(\boldsymbol{F})$ is the volume change ratio. Furthermore, $\tilde{I}_{4}=\tilde{\boldsymbol{C}}: \vec{n}_{0} \vec{n}_{0}$ is the isochoric fourth invariant where $\vec{n}_{0}$ is the fiber direction vector in the reference configuration with unit length; $k_{1}$ is the scalar fiber stiffness, and $\kappa$ is the dispersion of the fiber orientations around the preferred fiber direction $\vec{n}_{0}$. The two limits of $\kappa$ are 0 for fully aligned fibers (i.e., transverse isotropy) and $\frac{1}{3}$ for randomly oriented fibers (i.e., isotropy). Therefore, the value of $\kappa$ is related to the degree of anisotropy. By means of the Macaulay brackets $\langle\cdot\rangle$, the fibers contribute only in tension and not in compression, as $\langle\tilde{E}\rangle$ becomes 0 if $\tilde{E}$ is negative. The fiber contribution to the stiffness is assumed linear (i.e., $k_{2} \rightarrow 0$ ) (Cloots et al. 2011). Viscoelasticity is added by using:

$\boldsymbol{S}(t)=\int_{0}^{t}\left[M_{\infty}+\sum_{i} M_{i} \exp \left(-\frac{t-\tau}{\tau_{i}}\right)\right] \frac{\partial \boldsymbol{S}^{e}}{\partial \tau} \mathrm{d} \tau$, 
where $M_{\infty}+\sum_{i} M_{i}=1, \tau$ is a time variable that runs from the initial time up to the current time $t, S(t)$ is the current deviatoric second Piola-Kirchhoff stress tensor, $S^{e}$ is the deviatoric elastic second Piola-Kirchhoff stress tensor as derived from the non-volumetric part of Eq. 1 (i.e., the first and third term on the right side), $M_{\infty}$ is the long-term parameter recovering the role of the material parameters $G$ and $k_{1}$ in the limit, $M_{i}$ are the relaxation parameters of the viscoelastic modes and $\tau_{i}$ are the time constants. The volumetric behavior is assumed to be independent of time.

Several studies in literature indicate that brain tissue behaves anisotropically (Arbogast et al. 1995; Arbogast and Margulies 1998; Hrapko et al. 2008; Nicolle et al. 2004, 2005; Ning et al. 2006; Prange et al. 2000; Prange and Margulies 2002). However, rheological measurements in literature show a variation in stiffness of brain tissue of several orders of magnitude due to a strong sensitivity to many testing conditions (Hrapko et al. 2008). Considering these variations in stiffness, the orientation dependence used in this study was obtained from the experimental study of Ning et al. (2006) on the brainstem of a 4-week-old pig, but the effective absolute stiffness was chosen to correspond with that of the original, validated, head model (Kleiven 2007). It is noted that the concentration of strain in the CVE (which is described further on) relative to the strain applied to it depends on the relative amount of anisotropy within the material and not on the absolute stiffness of it. The brain tissue material properties of the current study are given in Table 1 . Since the original head model has been validated with isotropic mechanical behavior only, the mechanical response of the original and the extended model are expected to be similar in case the extended model would contain only isotropically oriented fibers (i.e., $\kappa=\frac{1}{3}$ ) in the brain tissue. The ratio of the parameter values for $G$ and $k_{1}$ is assumed to be the same as the ratio in the study by Ning et al. (2006) although the absolute parameter values are not taken from this study. The fiber orientation is fully uniaxially in the corpus callosum (lateral orientation), the brainstem (inferior-posterior orientation) and the spinal cord (inferior-posterior orientation), which will result in anisotropic mechanical behavior. Although the degree of anisotropy varies throughout the brain, the fiber orientation in the remaining part of the brain tissue is modeled fully isotropic, because it is assumed that due to heterogeneities and/or lower levels of axonal alignment, the anisotropy in those other regions is much less important than for the brainstem and the corpus callosum. In the original isotropic head model, the brainstem has a higher stiffness than the remaining brain tissue, which is here automatically accounted for by the increased stiffness from the fiber contribution in the inferior-posterior orientation. The bulk modulus of brain tissue is $2.1 \mathrm{GPa}$ (McElhany et al. 1976), but to prevent volumetric locking of the elements, a lower value of $50 \mathrm{MPa}$ is used, which is still much higher than the shear modulus. For
Table 1 Material properties of the brain tissue in the head model

\begin{tabular}{lll}
\hline$G(\mathrm{~Pa})$ & 1,214 \\
$k_{1}(\mathrm{~Pa})$ & 11,590 & \\
$M_{1}$ for $\tau_{1}=10^{-6} \mathrm{~s}$ & 0.7685 & \\
$M_{2}$ for $\tau_{2}=10^{-5} \mathrm{~s}$ & 0.1856 & \\
$M_{3}$ for $\tau_{3}=10^{-4} \mathrm{~s}$ & 0.0148 & \\
$M_{4}$ for $\tau_{4}=10^{-3} \mathrm{~s}$ & 0.0190 & \\
$M_{5}$ for $\tau_{5}=10^{-2} \mathrm{~s}$ & 0.0026 & Anisotropic parts \\
$M_{6}$ for $\tau_{6}=10^{-1} \mathrm{~s}$ & 0.0070 & 0 \\
$M_{\infty}$ & 0.0025 & \\
\hline & & \\
\hline
\end{tabular}

compact bone tissue, a Young's modulus of $15 \mathrm{GPa}$ is used, whereas for porous bone and the neck bone, this value is $1 \mathrm{GPa}$. The dura mater, the falx, and the tentorium are modeled with a Young's modulus of $31.5 \mathrm{MPa}$ and the pia mater with 11.5 MPa. Furthermore, a sliding interface with a friction coefficient of 0.2, as proposed by Miller et al. (1998), is used between the dura and the skull.

The loading conditions of the head model are based on a reconstruction case of an accident in the American National Football League with case numbers 57H2 (Viano et al. 2005; Kleiven 2007). This case is involved with loss of consciousness, which is often related to brainstem injury (Shaw 2002). In Fig. 3, the acceleration of the head model is shown.

\subsection{Critical volume element}

The CVE is developed using the FE code Abaqus 6.102 (Abaqus 2010). As opposed to the head model, the CVE is analyzed with a quasi-static computation with an implicit integration scheme. It contains 27,960 eightnode reduced integration hexahedral elements. The threedimensional geometry of the CVE is based on pathological observations for axonal injury. It contains a spherical inclusion with a cross-sectional diameter of 0.16 relative to the outer dimensions of the CVE (e.g., inclusion diameter is $8 \mu \mathrm{m}$ and outer dimensions are 50 by $50 \mu \mathrm{m}^{2}$ ). The material surrounding the inclusion is assumed to consist of axons only. Note that for a given axonal orientation field, the size of the inclusion does not affect the strains in the CVE (Cloots et al. 2012). The inclusion and the surrounding tissue are assumed to be fully compatible at the interface. The tissue, consisting of axons, is modeled with a continuum approach. In line with the head model used at the macroscopic level, an anisotropic Holzapfel-Gasser-Ogden model (Gasser et al. 2006) is used with the same properties as the head model. Note that volume associated to these critical locations is statistically small, so 

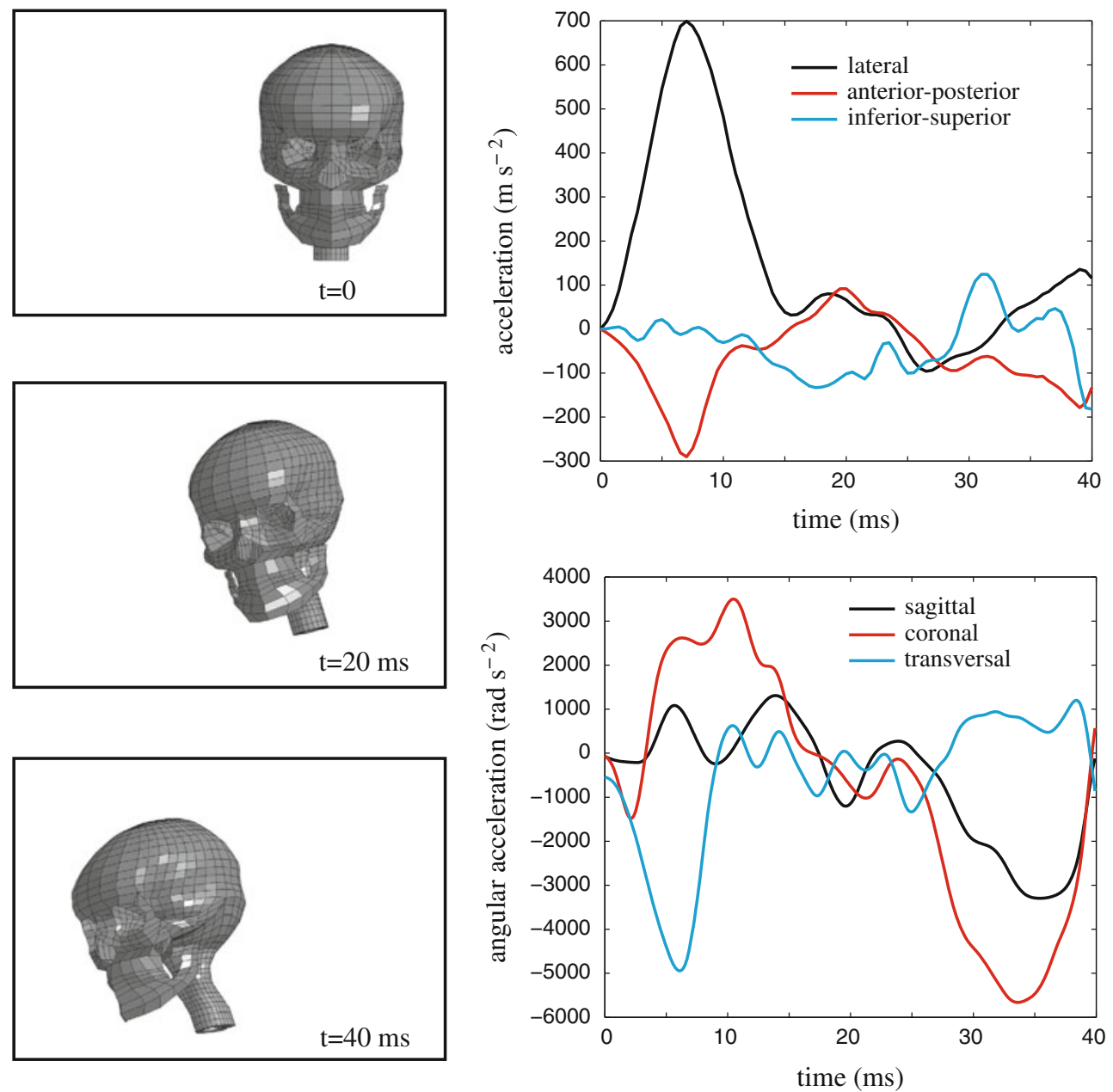

Fig. 3 Head model loading condition based on the reconstruction of a struck sports player [case number 57H2 (Viano et al. 2005)]

that the average tissue properties at the microstructural level correspond with those at the macroscopic level. Furthermore, the neurofilaments causing the anisotropic behavior are characterized by a much smaller length scale than the macro-level head model as well as the micro-level CVE. The same material model can therefore be used in the head model and the CVE without violating the separation of length scales. In order to obtain the axonal strains, each material point of the CVE has a specific orientation representing the local axonal orientation, which is depicted in Fig. 4. The CVE simulations are conducted for material points (i.e., integration points in the FE discretization) of the brainstem and the corpus callosum in the head model and therefore, the main axonal direction in the CVE is aligned with the local axonal direction of the brainstem and the corpus callosum in the head model.

The behavior of the inclusion is also described with the Holzapfel-Gasser-Ogden model, but with isotropically oriented fibers (i.e., $\kappa=\frac{1}{3}$ ). To investigate the influence of the inclusion stiffness, three different values of the elastic parameters of the inclusion relative to those of the brain tissue are used: (A) equal to those of the isotropic brain tissue, (B) three times stiffer, and (C) ten times stiffer. The bulk modulus is constant over the entire CVE and is equal to that of the head model. The material properties are shown in Table 2.

The loading conditions of the CVE are obtained from the deformation gradient tensor in the corresponding material point in the brain tissue as predicted with the head model and imposed by means of periodic boundary conditions (Kouznetsova et al. 2001). The displacement vector $\vec{u}$ of a corner node $c_{i}$, as shown in Fig. 5, is calculated from the global deformation gradient tensor $\overline{\boldsymbol{F}}$, which is obtained from the Green-Lagrange strain tensor in the head model, through:

$\vec{u}_{\mathrm{c}_{i}}=(\overline{\boldsymbol{F}}-\boldsymbol{I}) \cdot \vec{x}_{0 \mathrm{c}_{i}}$,

in which $\vec{x}_{0}$ is the initial position vector and $\boldsymbol{I}$ is the unit tensor. Equation 4 is prescribed at the corner nodes $c_{1}, c_{2}$, $\mathrm{c}_{4}$, and $\mathrm{c}_{5}$. The nodal displacement vectors of the remaining parts of the boundary are tied as follows: 


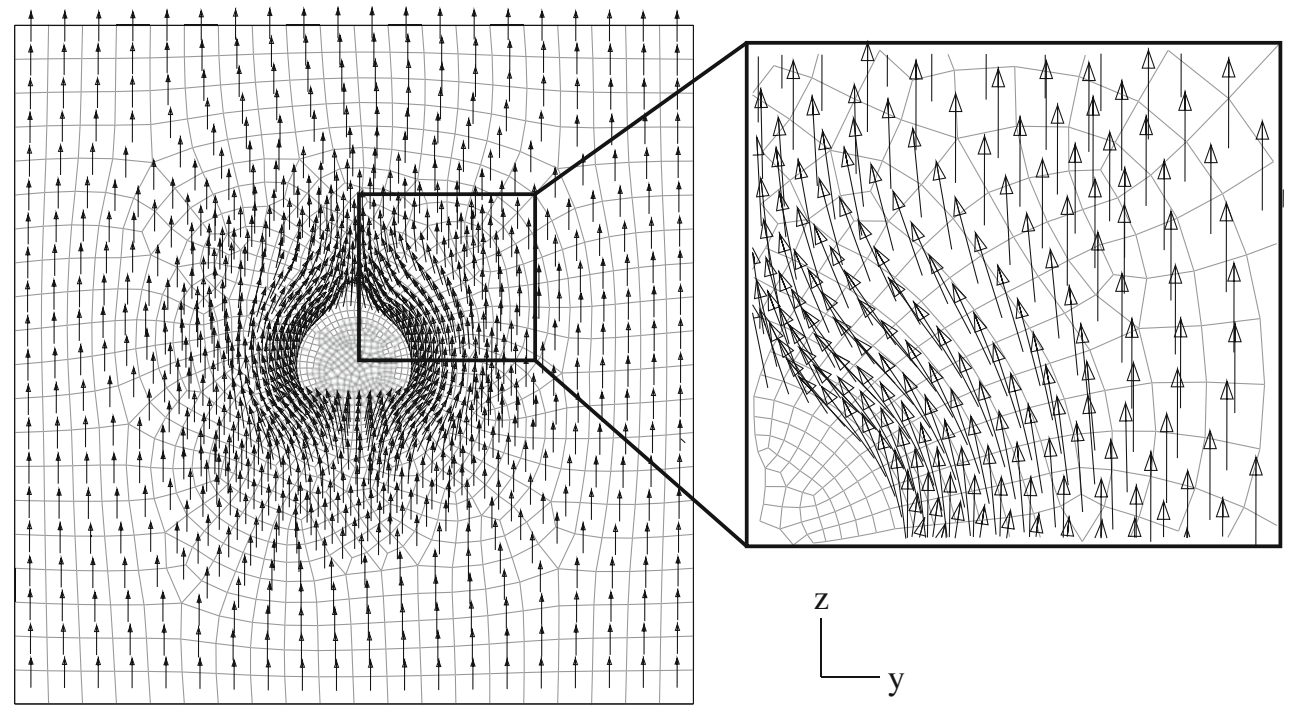

Fig. 4 Cross-section of the CVE with the spatial discretization and the axonal orientation in each element of the part that consists of axons

Table 2 Material properties of the CVE

$\vec{u}_{\Gamma_{5678}}-\vec{u}_{\Gamma_{1234}}=\vec{u}_{\mathrm{c}_{5}}-\vec{u}_{\mathrm{c}_{1}}$,

$\vec{u}_{\Gamma_{2673}}-\vec{u}_{\Gamma_{1584}}=\vec{u}_{\mathrm{c}_{2}}-\vec{u}_{\mathrm{c}_{1}}$,

$\vec{u}_{\Gamma_{4378}}-\vec{u}_{\Gamma_{1265}}=\vec{u}_{\mathrm{c}_{4}}-\vec{u}_{\mathrm{c}_{1}}$,

where $\Gamma_{j k l m}$ denotes the faces of the model (see Fig. 5). As a result of these kinematical boundary conditions, antiperiodicity of the tractions is satisfied (Kouznetsova et al. 2001). This leads to a multi-scale coupling between the head model and the CVE, in which both volumetric and deviatoric deformations are transferred from the macro to the micro level.

\subsection{Anisotropic equivalent strain measure}

In the previous work (Cloots et al. 2012), an anisotropic equivalent strain measure $\bar{\varepsilon}^{\text {eq }}$ has been developed that represents the maximum axonal strain as a result of the tissue strain components $\bar{\varepsilon}_{i j}$ :

$$
\begin{aligned}
\bar{\varepsilon}^{\mathrm{eq}}= & {\left[F\left(\bar{\varepsilon}_{y y}-\bar{\varepsilon}_{z z}\right)^{2}+G\left(\bar{\varepsilon}_{z z}-\bar{\varepsilon}_{x x}\right)^{2}+H\left(\bar{\varepsilon}_{x x}-\bar{\varepsilon}_{y y}\right)^{2}\right.} \\
& \left.+2 L \bar{\varepsilon}_{y z}^{2}+2 M \bar{\varepsilon}_{z x}^{2}+2 N \bar{\varepsilon}_{x y}^{2}\right]^{\frac{1}{2}}+I \bar{\varepsilon}_{x x}^{d}+J \bar{\varepsilon}_{y y}^{d}+K \bar{\varepsilon}_{z z}^{d},
\end{aligned}
$$

This equivalent strain measure accounts for the orientation dependence governing the contribution of tissue deformations to axonal stretching. The coefficients of this equivalent strain were obtained from a CVE on which isochoric uniaxial and biaxial deformations in all loading directions were applied. Deviatoric strains were used in Cloots et al. (2012), since injury due to axonal straining is considered to be caused mainly by non-volumetric global deformations as a consequence of the high bulk modulus of the brain tissue. For this reason, the equivalent strain measure of Eq. 8 is a function of deviatoric strains only. The values are given in Table 3, and they are obtained with a CVE with a spherical inclusion, in which the main axonal direction is in the $y$-direction. To simplify the analysis and eliminate the coupled solution at two scales, the equivalent strain measure can be used to replace the CVE as depicted in Fig. 6. In the current study, the predictions based on this equivalent strain measure will be compared with the maximum axonal strain values obtained from the CVE simulations.

\section{Results}

In Fig. 7, a strain field is shown for the head model, from which it is clear that elevated (macroscopic) strain levels up to 0.28 occur, especially around the brainstem. For this particular situation, in which loss of consciousness was observed, 


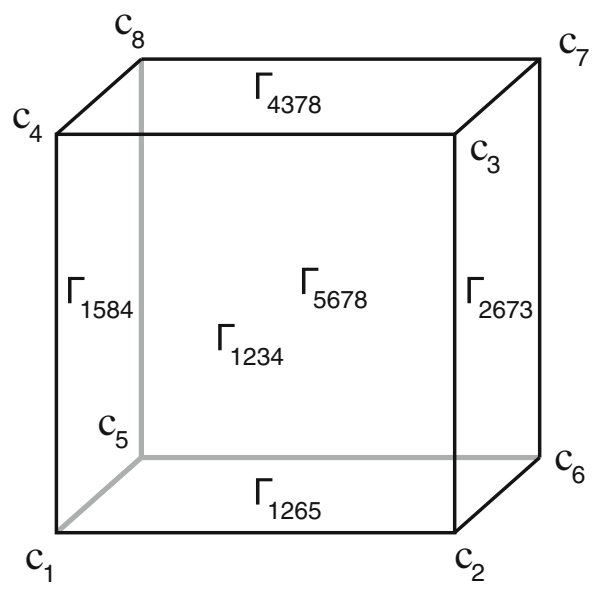

(a)

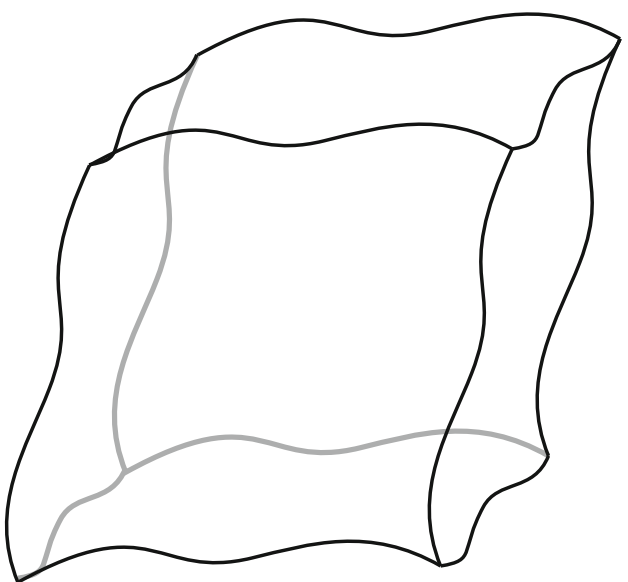

(b)

Fig. 5 CVE with a labeling of the corners and faces in the undeformed state and $\mathbf{b}$ deformed model with periodic boundary conditions

Table 3 The values of the coefficients of the equivalent strain measure in Eq. 8 as obtained from CVE calculations (Cloots et al. 2012)

\begin{tabular}{llllllllll}
\hline Coefficient & $F$ & $G$ & $H$ & $L$ & $M$ & $N$ & $I$ & $J$ & $K$ \\
\hline Value & 0.163 & -0.056 & 0.163 & 0.119 & 0.051 & 0.119 & 0.000 & 0.707 & 0.000 \\
\hline
\end{tabular}

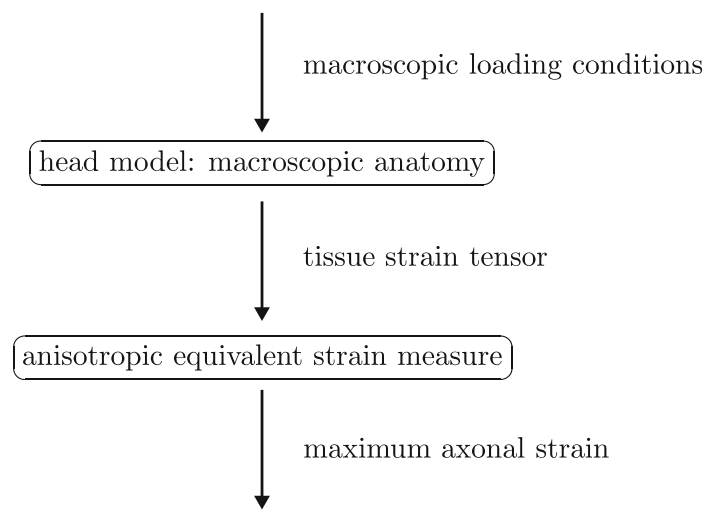

Fig. 6 Schematic representation of the implementation of the anisotropic equivalent strain measure

this indicates that the brainstem is a vulnerable part for injury in case an injurious mechanical load occurs.

The maximum axonal strains obtained from a CVE simulation as a function of the tissue strain in a specific material point in the brainstem and the corpus callosum are plotted in Fig. 8. No clear relation between the axonal strain and the maximum principal tissue strain is observed, both for the simulations with and without an inclusion. Following the trajectory in time, it can be noticed that two peak values of the maximum principal logarithmic tissue strain are reached in the brainstem as well as the corpus callosum. For these two peak values, however, the ratio between the tissue strain and the maximum axonal strain is different. The presence of an

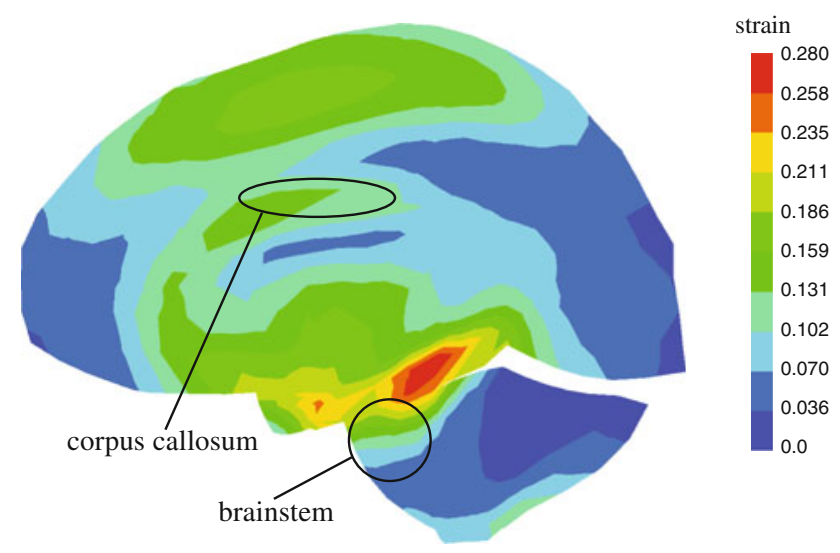

Fig. 7 Maximum principal logarithmic strain of a sagittal crosssection of the head model at $37 \mathrm{~ms}$

inclusion leads to increased local maximum axonal strains in the tensile regime. Furthermore, the relation between the axonal strain and the tissue strain is also dependent on the angle $\varphi$ (between the maximum principal loading direction of the tissue and the main axonal direction), which is most pronounced in the trajectory without an inclusion. For this case, when the angle $\varphi$ approaches $90^{\circ}$, a compressive axonal strain is found, whereas the maximum principal tissue strain is positive.

The influence of the stiffness of the blood vessel on the maximum local axonal strain is displayed in Fig. 9. The inclusions $\mathrm{A}, \mathrm{B}$, and $\mathrm{C}$ have material properties that are nearly 

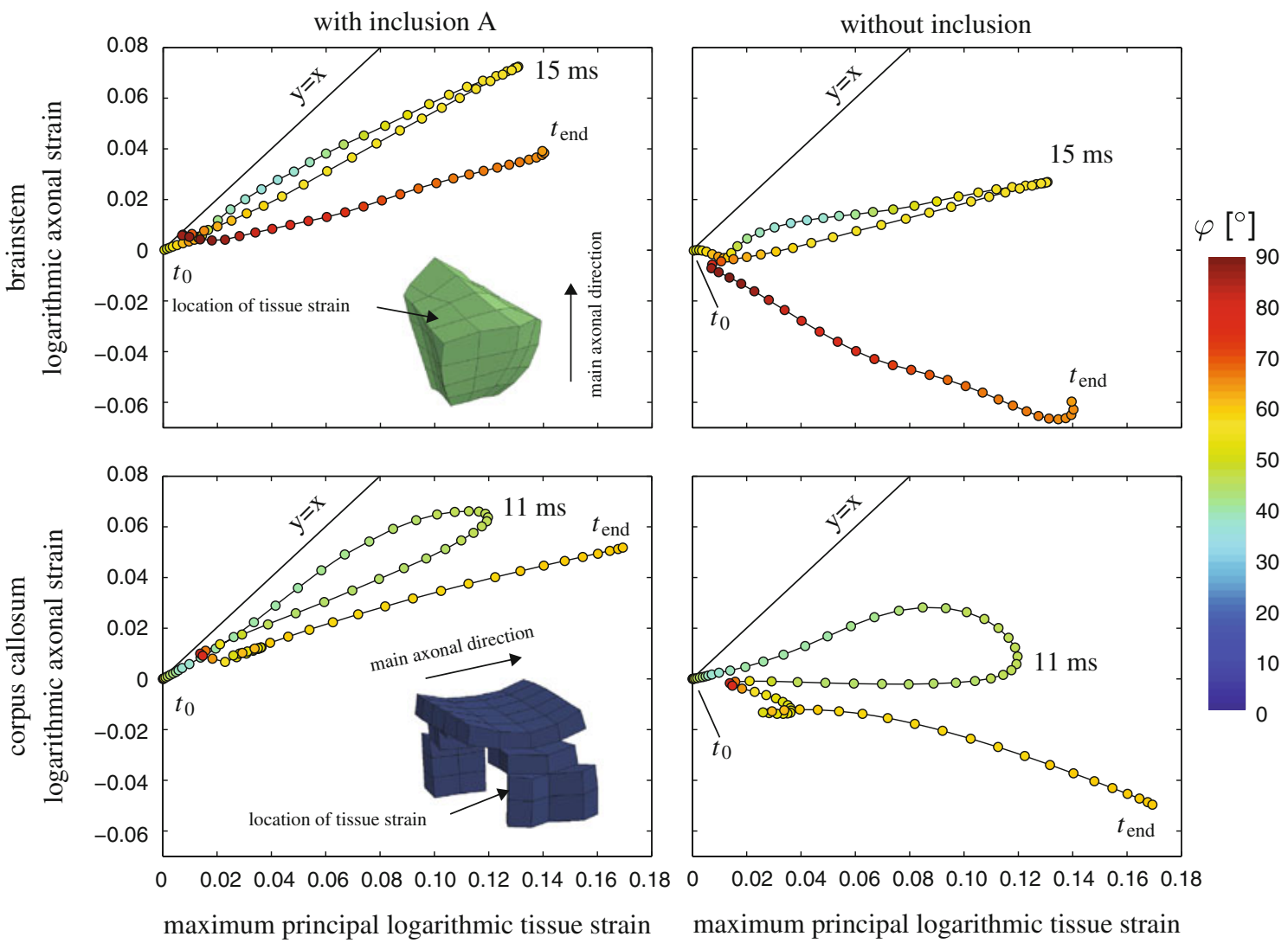

corpus callosum with an arrow indicating the material point of which

Fig. 8 Time trajectory of the maximum axonal strain in the CVE versus the maximum principal tissue strain from $t_{0}$ to $t_{\text {end }}$ during the mechanical loading for one point in the brainstem and one point in the corpus callosum in the head model. The inset shows the brainstem and the the tissue loading is obtained. The colors refer to the angle $\varphi$ between the maximum principal loading direction of the tissue and the main axonal direction

equal to the brain tissue, three times higher, or ten times higher, respectively. The only difference is that for all inclusions $\kappa=\frac{1}{3}$ as opposed to $\kappa=0$ for the anisotropic brain tissue. Although the overall shape of the trajectory remains similar for all three cases, some important differences are present. At $15 \mathrm{~ms}$, the axonal strain values are lower for an increased stiffness of the inclusion, whereby an increase with a factor 10 of the inclusion stiffness leads to a decrease of the maximum axonal strain by $\frac{1}{3}$. At time $t_{\text {end }}$, however, the effect of the stiffness is different, where the axonal strains for inclusion $\mathrm{A}$ and $\mathrm{C}$ are similar and those for inclusion $\mathrm{B}$ are lower. Because the surrounding brain tissue is anisotropic and the inclusion is isotropic, the stiffness of the inclusion relative to the brain tissue depends also on the loading direction, which causes the dissimilarities between $15 \mathrm{~ms}$ and $t_{\mathrm{end}}$.

Figure 10 shows the axonal strain field of the CVE with inclusion A obtained from the material point in the brainstem (used for Fig. 8) during the injurious load case at $15 \mathrm{~ms}$. Adjacent to the inclusion, strain concentrations are present at the

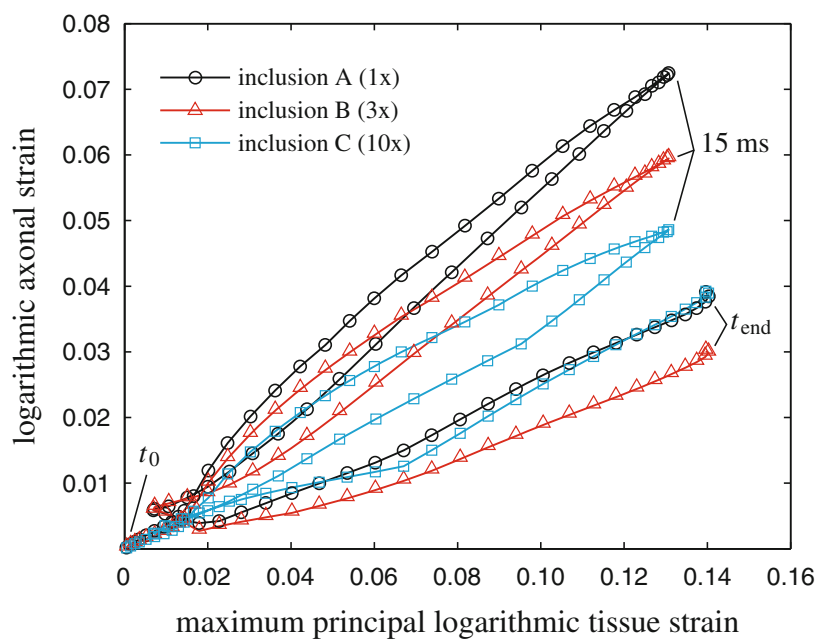

Fig. 9 Trajectories of the maximum axonal strain in the CVE versus the maximum principal tissue strain from $t_{0}$ to $t_{\text {end }}$ during the mechanical loading for the same location in the brainstem in the head model with three different degrees of stiffness of the inclusion 


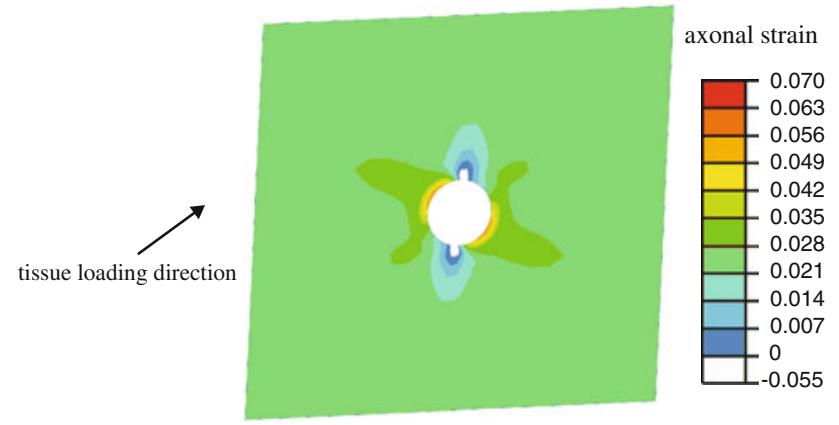

Fig. 10 Cross-section of the axonal strain field of the CVE at $t=15 \mathrm{~ms}$ in the brainstem. For clarity, the strain field of the inclusion is not depicted

location where the local axonal orientation is most aligned with the tissue loading direction. One can also notice that the axonal strain values more distant from the inclusion are in agreement with the strain values of the case without an inclusion (see Fig. 8).

When the axonal strain is plotted against the maximum principal tissue strain for all elements of the brainstem in the head model at each time step (see Fig. 11), it is observed that overall the maximum principal tissue strain is an overprediction for the maximum axonal strain in the CVE. The amount of this overprediction is partially related to the angle $\varphi$, where higher angles generally result in a larger overprediction. The results of the simulations with an inclusion show a good correlation between axonal and tissue strains for the range of angles $\varphi$ from $0^{\circ}$ to about $40^{\circ}$. However, still many local values deviate from these general observations. This indicates that the maximum principal strain observed in the tissue of the head model is not able to predict strains occurring at the axonal level due to the influence of axonal orientation. Furthermore, the inclusion causes the axonal strains to be larger than without an inclusion for the same tissue strains. Moreover, whereas compressive axonal strains are occurring for the situation without an inclusion, they are only in the tensile regime for the situation with an inclusion. This is caused by the local deviation of axonal orientation near the inclusion due to which at least some of the axons are always fully or partially aligned with the tissue loading direction, which is not the case without an inclusion.

In a first step toward including the effects of axonal orientation, the (macroscopic) tissue strains in the main axonal direction are considered, for which it should be noted that this is the same as the axonal strain for a region without an inclusion. Figure 12 depicts the axonal strain of the CVE with an inclusion versus the tissue strain of the head model in the main axonal direction for all elements of the brainstem. For a situation without an inclusion, the same values would be plotted on both axes and therefore the line $y=x$ can be interpreted as the axonal strain value for a situation without an inclusion. It can be noticed that the inclusion causes the axonal strains to increase, in particular when the angle between the loading direction and the main axonal direction is large and the tissue strain is positive. Nevertheless, several instances with a smaller angle still lead to high axonal strains indicating that the tissue strain in the main axonal direction is not able to predict the local axonal strain accurately in case of an inclusion. Only for lower values of $\varphi$, the axonal strain shows a close agreement with the tissue strain in the main axonal direction. Furthermore, it is observed that also negative tissue strains in the main axonal direction lead to axonal stretching. The general observation is that the tissue strain in the main axonal direction is an underprediction of the maximum axonal strain in the presence of an inclusion and the amount of underprediction depends partially on the angle $\varphi$.

In Fig. 13, tissue and axonal strains obtained in the brainstem and the corpus callosum are plotted as a function of time. The maximum axonal strains obtained from the CVE simulations are between the maximum principal tissue strain and the tissue strain in the main axonal direction during the entire simulation time. In the brainstem, the time-averaged relative differences of the maximum principal tissue strain, the tissue strain in the main axonal direction, and the anisotropic equivalent strain with respect to the maximum axonal strain in the CVE are $2.05 \pm 0.19,0.39 \pm 0.11$, and $1.05 \pm 0.064$ (mean \pm standard deviation), respectively. In the corpus callosum, the relative differences are $2.23 \pm 0.63,0.33 \pm 0.17$ and $1.05 \pm 0.059$, respectively. The anisotropic equivalent strain agrees therefore the most with the maximum axonal strain in the CVE in terms of both the relative mean differences and the smallest standard deviations. For this loading case, the profile of the maximum axonal strain in the CVE is similar to that of the maximum principal tissue strain for the brainstem. However, the profiles of the remaining tissue strains, in particular the tissue strain in the main axonal direction in the corpus callosum, deviate from the maximum axonal strain in the CVE (i.e., the standard deviations of the relative differences are large with respect to their mean values). This indicates that axonal strains cannot be generally obtained from the maximum principal tissue strain or the tissue strain in the main axonal direction. Furthermore, it can be noticed that the anisotropic equivalent strain, which is obtained directly from the tissue in the macroscopic head model, agrees well with the maximum axonal strain obtained from the CVE simulations.

For a more in-depth comparison between the CVE simulations and the anisotropic equivalent strain measure, the maximum axonal strain of the CVE simulations is plotted versus the anisotropic equivalent strain in Fig. 14 for all material points of the brainstem. It can be observed that all dots are close to the solid black line that represents an exact agreement between the axonal strain and the equivalent strain. 

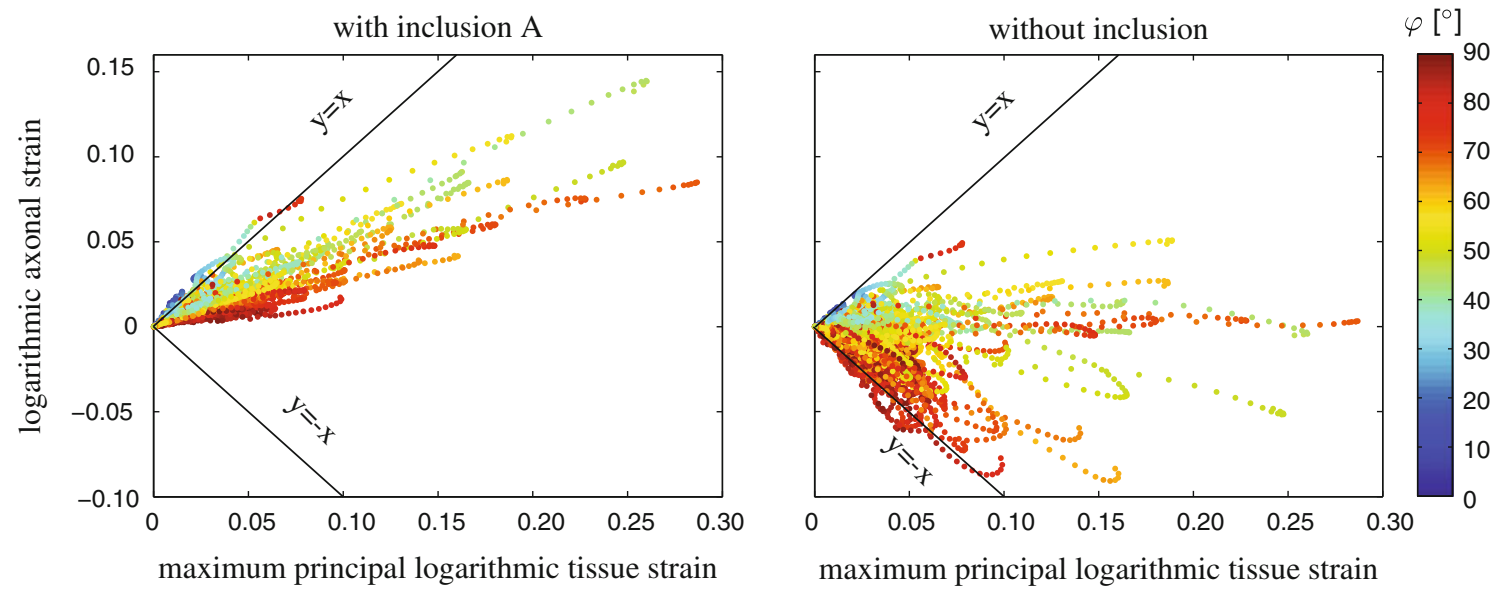

Fig. 11 Maximum axonal strain in the CVE versus the maximum principal tissue strain at each time step during the mechanical loading for all elements of the brainstem in the head model

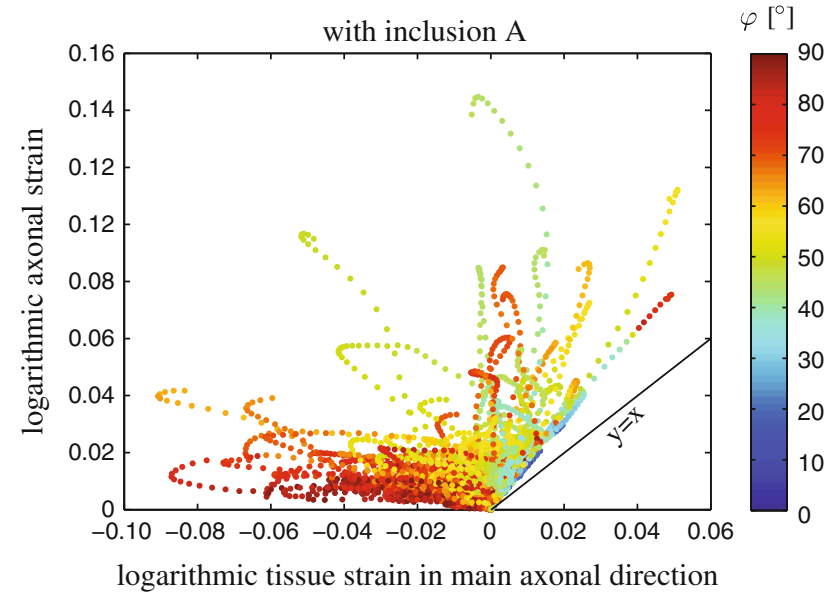

Fig. 12 Maximum axonal strain in the CVE with an inclusion versus the tissue strain in the main axonal direction at each time step during the mechanical loading for all elements of the brainstem in the head model
For large angles of $\varphi$, the outcome of the CVE is generally higher than the equivalent strain, whereas for small angles, it is generally the opposite. The relative differences are $20 \%$ or less for equivalent strains higher than 0.06 and less than $7 \%$ for equivalent strains above 0.10 . The overall $R^{2}$ value is 0.98 . It is clear that the anisotropic equivalent strain is a better predictor for axonal strains as obtained from the CVE than the maximum principal tissue strain (see Fig. 11, lefthand side) and the tissue strain in the main axonal direction (see Fig. 12).

The strain fields of the brainstem are shown in Fig. 15 at $37 \mathrm{~ms}$, when the highest strain values have been reached. The anisotropic equivalent strain is not only lower than the maximum principal tissue strain, but also the strain concentrations are in different points for either the anisotropic equivalent strain or the maximum principal tissue strain. Furthermore, the material point for which the highest equivalent strain in
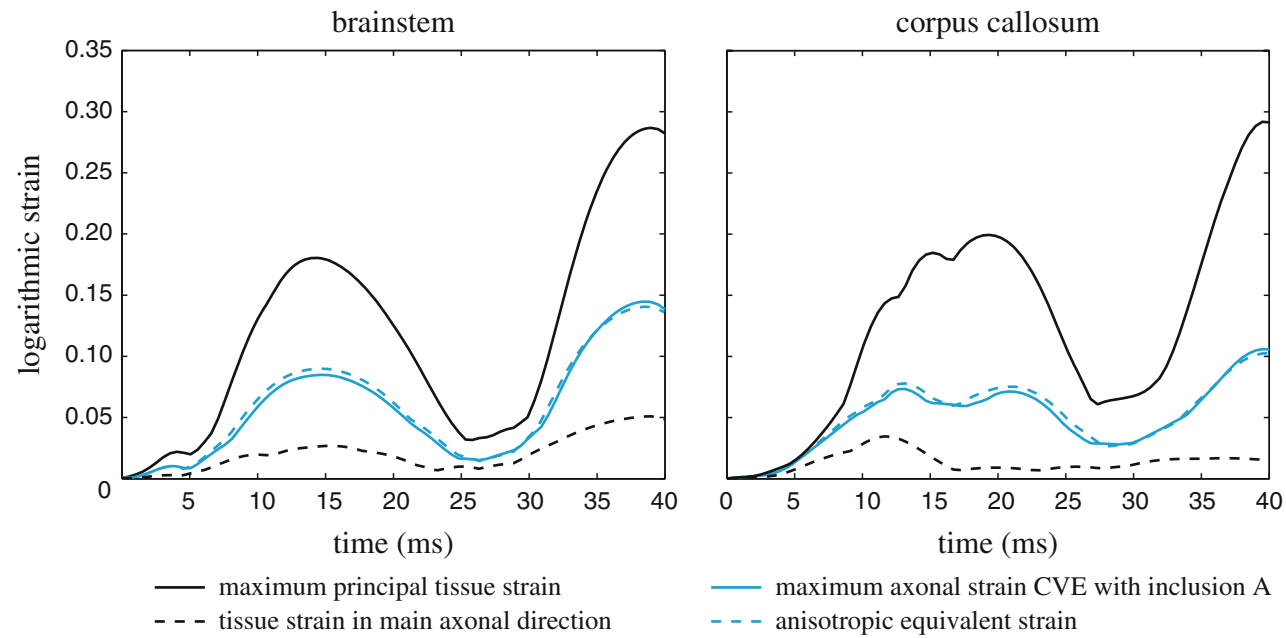

Fig. 13 Maximum values of the tissue and the axonal strains versus time in the brainstem and the corpus callosum 


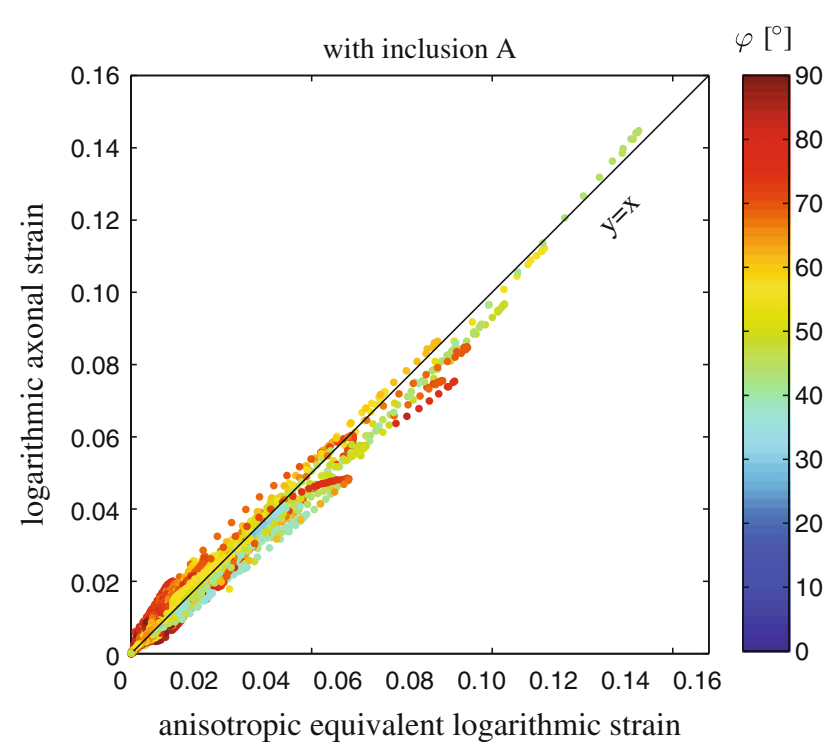

Fig. 14 Maximum axonal strain in the CVE with an inclusion versus the anisotropic equivalent strain of the tissue at each time step during the mechanical loading for all elements of the brainstem in the head model

the brainstem is computed is the same as that obtained by means of the CVE simulations.

\section{Discussion}

In this study, a multi-scale approach was used with a macroscopic FE head model to simulate the tissue response to mechanical loading and a micro-mechanical FE model of a CVE to obtain the local axonal strains due to the heterogeneities at the cellular level, which are assumed to be the cause of the discrete local axonal impairments in case of DAI within the brainstem and the corpus callosum. The strain values of the CVE and the FE head model have no trivial correlation. The maximum principal tissue strain in the $\mathrm{FE}$ head model is shown to be an overprediction for the maximum axonal strain in the CVE. In a study using the same FE head model, but with isotropic viscoelastic material behavior, performed by Kleiven (2007), it was shown that loading conditions associated with concussion result in predictions of relatively high strain levels that are on the same level as suggested for DAI (Bain and Meaney 2000), even though the mechanical behavior for brain tissue in the model corresponds with the effective shear modulus of approximately $10 \mathrm{kPa}$ at $80 \mathrm{~Hz}$ found for brain tissue in vivo by (McCracken et al. 2005) using magnetic resonance elastography. The lack of correlation between the tissue strain and the diagnosis of concussion in the study by Kleiven (2007) might be explained by the overprediction of tissue strains for the interpretation of the maximum axonal strain, which is shown in the current study. Furthermore, the tissue strain in the main axonal direction in the head model is shown to be an underprediction for the maximum axonal strain in the CVE. More importantly, the amount of over- and underprediction depends on the microstructure and the loading direction, which indicates that criteria for DAI should be developed taking into account the effects of the cellular level. In addition to the multi-scale method, an anisotropic equivalent strain measure (Cloots et al. 2012) was implemented in the FE head model to estimate axonal strains through a single-scale analysis. The equivalent strain was shown to be in close agreement with the outcome of the CVE simulations, whereas it requires only tissue strains predicted with a macroscopic head model and does not involve a multi-scale analysis. Therefore, since the CVE in this study is modeled with viscoelastic, anisotropic tissue behavior and the equivalent strain measure was originally developed for a hyperelastic, anisotropic tissue behavior, it
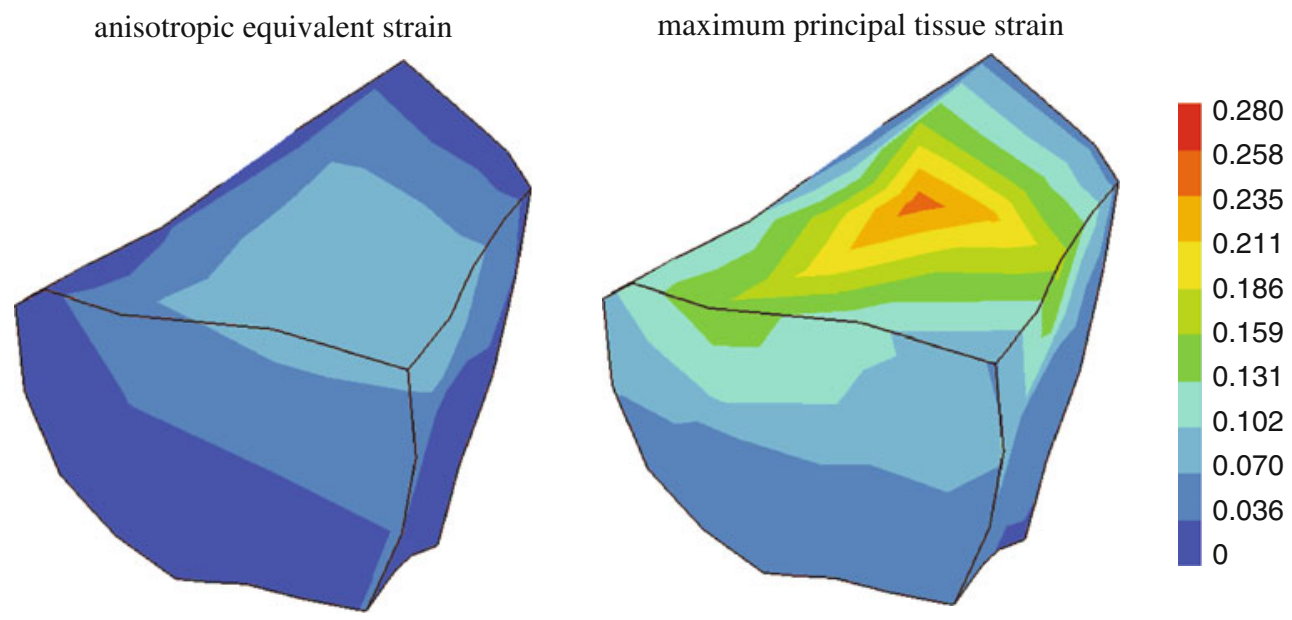

Fig. 15 Field plots of the strain values in the brainstem at $37 \mathrm{~ms}$ for the injurious loading case $57 \mathrm{~h} 2$ 
can be concluded that viscoelasticity has no significant influence on the micro-level strains, unlike the tissue-level strains.

The FE head model has a geometry that distinguishes several parts of the brain (e.g., cerebral cortex, corpus callosum, brainstem). Although it does not include a detailed geometry (e.g., the folding structure of the cerebral cortex), the influence of these details is assumed to be local and therefore does not affect the tissue deformations of the brainstem and the corpus callosum. Although in reality, the brainstem would be more cylindrically shaped than in the model, the total volume is close to MRI volumetric measurements of the brainstem from healthy volunteers (Kleiven 2007).

The loading conditions of the FE head model are based on the reconstruction of a specific injurious sports accident using HIII dummies (Kleiven 2007). Because of this, only the motion of the head could be applied directly to the head. Therefore, the possibly important influence of spinal cord bending could not be included in the analysis, which might affect the strain levels in the brainstem. Nevertheless, since this study is concerned with the maximum local axonal strains with respect to the tissue strains, the conclusions drawn are expected to remain valid for slightly different global mechanical loads.

The material properties of the head model were originally isotropic (Kleiven 2007), but for this study, the behavior was extended with anisotropy, because several studies have shown that areas of the brain with aligned axons reflect an anisotropic material behavior (e.g., Arbogast and Margulies 1998, 1999; Prange and Margulies 2002; Nicolle et al. 2005; Ning et al. 2006; Hrapko et al. 2008). In the original isotropic head model, the brainstem was modeled with stiffer material properties than the remaining brain tissue. In the current study, it is assumed that the stiff nature of the brainstem is justified only in the main axonal direction due to the anisotropic behavior. Therefore, the current anisotropic head model contains the same material properties for the entire brain tissue, except for the fiber orientation distribution (i.e., either uniaxially aligned or randomly oriented). Although the degree of anisotropy varies throughout the brain and is simplified in the model (i.e., only brainstem and corpus callosum are modeled as anisotropic), it is expected that the effect of including a higher level of anisotropy elsewhere in the brain on the outcome of this study would be relatively small.

In a previous study (Cloots et al. 2011), it was shown that anisotropy at the tissue level causes the strains to become smaller in the main axonal direction, but larger in other directions for the same stress levels. For a homogeneous axonal alignment (e.g., without the presence of an inclusion), this would also lead to reduced axonal strains, even for the higher strain levels in other directions than the main axonal direction, since then the loading direction is not aligned with the axons anymore. However, when the axons have to deviate for an inclusion, partial alignment exists for a wider range of loading directions compared to the one without an inclusion. Hence, these axons have a higher strain locally caused by anisotropic material behavior. Nevertheless, the focus of this study is on the differences between the tissue-level strains and the axonal strains.

The CVE has a geometry with a simplified inclusion, which has a spherical shape that represents a cell body. In a parallel study (Cloots et al. 2012), also a cylindrical inclusion was used that represents a blood vessel. It was found that the most critical configuration of the cylindrical inclusion (i.e., main axonal direction about $30^{\circ}$ with respect to the long axis of the cylinder) resulted in the same strain levels as with the spherical inclusion. Because blood vessels inside the brain are oriented rather randomly, it is assumed that this most critical configuration likely exists. The similar influence of the spherical and the most critical cylindrical configuration on the axonal strains originate from the fact that the local axonal orientation with respect to the loading direction has a greater influence on the axonal strain than the actual shape of the inclusion. Therefore, it is assumed that the CVE with a spherical inclusion does not only adequately represent the case of a cell body, but also the case of a network of blood vessels. The stiffness of the inclusion relative to the surrounding axons has an influence on the axonal strain levels relative to the tissue strain level, but the overall relation between tissue and axonal strains remains similar.

Currently, the CVE is coupled only to the brainstem and the corpus callosum of the head model. Because also other parts of the brain can be involved with DAI, it may be necessary to couple these parts as well. In general, axons in other parts of the brain are less aligned, which will probably result in a different relation between the axonal strains and the tissue strains than for the brainstem and the corpus callosum. Also, different regions of the brain could have different tolerance criteria at the cellular level, which has been shown in a study by Elkin and Morrison (2007). Furthermore, variations in tissue densities are an important aspect of axonal injury in some parts of the brain (Gaetz 2004). This indicates there are more microstructural aspects that might play a role in cellular-level injury and that have not been included in the CVE used in the current study. Furthermore, the brainstem and the corpus callosum have a relatively simple geometry, whereas other parts of the brain might need more detailed geometries of the FE head model to obtain realistic tissue deformations. A more extended implementation of anisotropy could therefore be realized in a detailed FE head model created by automatic segmentation and meshing based on medical images such as the model created by Ho et al. (2009) or by Chen and Ostoja-Starzewski (2010). Simulations with the detailed model of Ho et al. showed a similar correlation with the localized brain motion experiments of Hardy et al. (2001), as observed for the model used in the present study. 


\section{Conclusion}

This study shows that in the presence of microstructural obstructions that cause a deviation of axonal pathways, within the brainstem and the corpus callosum, axonal strains deviate from the maximum principal tissue strains as well as from tissue strains in the main axonal direction that are predicted in an FE head model, where the tissue strains are an overprediction or an underprediction of the maximum axonal strains, respectively. The main observation, however, is that tissue strains do not consistently scale with the maximum axonal strain, since the amount of over- and underprediction depends on the loading direction relative to the main axonal direction. This is caused by the local heterogeneities at the cellular level, which are local axonal orientation, difference in stiffness between the axons and the inclusion, and the anisotropic material behavior of the axons. The latter factor also influences the tissue strain in the FE head model. Since DAI is a type of injury in which the mechanical load occurs at the head level and the actual injury occurs at the cellular level, a multi-scale method with an FE head model and a CVE is a promising approach to obtain cellular-based injury criteria. Furthermore, a single-scale alternative to the multi-scale approach is the anisotropic equivalent strain measure, which can be used to obtain axonal strains directly from tissue-level strains. In combination with a critical value for injury, this measure can be used as an injury criterion.

Acknowledgments This work has been supported by the Dutch Technology Foundation STW, applied science division of NWO and the Technology Program of the Ministry of Economic Affairs, and the Swedish Research Council.

Open Access This article is distributed under the terms of the Creative Commons Attribution License which permits any use, distribution, and reproduction in any medium, provided the original author(s) and the source are credited.

\section{References}

Abaqus (2010) Abaqus 6.10 manual. Simulia, Providence

Alberts B, Bray D, Lewis J, Raff M, Roberts K, Watson JD (1994) Molecular biology of the cell, 3rd edn. Garland Publishing, New York

Arbogast KB, Margulies SS (1998) Material characterization of the brainstem from oscillatory shear tests. J Biomech 31:801-807

Arbogast KB, Margulies SS (1999) A fiber-reinforced composite model of the viscoelastic behavior of the brainstem in shear. J Biomech 32:865-870

Arbogast KB, Meaney DF, Thibault LE (1995) Biomechanical characterization of the constitutive relationship for the brainstem. In: Proceedings of the 39th Stapp car crash conference SAE, vol 952716, pp 153-159

Bain AC, Meaney DF (2000) Tissue-level thresholds for axonal damage in an experimental model of central nervous system white matter injury. J Biomech Eng 122:615-622
Bain AC, Raghupathi R, Meaney DF (2001) Dynamic stretch correlates to both morphological abnormalities and electrophysiological impairment in a model of traumatical axonal injury. J Neurotrauma 18:499-511

Cater H, Sundstrom L, Morrison III B (2006) Temporal development of hippocampal cell death is dependent on tissue strain but not strain rate. J Biomech 39:2810-2818

Chatelin S, Deck C, Renard F, Kremer S, Heinrich C, Armspach JP, Willinger R (2011) Computation of axonal elongation in head trauma finite element simulation. J Mech Behav Biomed 4:19051919

Chen Y, Ostoja-Starzewski M (2010) Mri-based finite element modeling of head trauma: spherically focusing shear waves. Acta Mech 213:155-167

Cloots RJH, Nyberg T, Kleiven S, van Dommelen JAW, Geers MGD (2011) Micromechanics of diffuse axonal injury: influence of axonal orientation and anisotropy. Biomech Model Mechanobiol 10:413-422

Cloots RJH, van Dommelen JAW, Geers MGD (2012) A tissue-level anisotropic criterion for brain injury based on microstructural axonal deformation. J Mech Behav Biomed 5:41-52

Cotter D, Mackay D, Chana G, Beasley C, Landau S, Everall IP (2002) Reduced neuronal size and glial cell density in area 9 of the dorsolateral prefrontal cortex in subjects with major depressive disorder. Cereb Cortex 12:386-394

Elkin B, Morrison III B (2007) Region-specific tolerance criteria for the living brain. Stapp Car Crash J 51:127-138

Elkin BS, Azeloglu EU, Costa KD, Morrison III B (2007) Mechanical heterogeneity of the rat hippocampus measured by atomic force microscope indentation. J Neurotrauma 24:812-822

Engel DC, Slemmer JE, Vlug AS, Maas AIR, Weber JT (2005) Combined effects of mechanical and ischemic injury to cortical cells: secondary ischemia increases damage and decreases effects of neuroprotective agents. Neuropharmacology 49:985-995

Floyd CL, Gorin FA, Lyeth BG (2005) Mechanical strain injury increases intracellular sodium and reverses $\mathrm{Na}^{+} / \mathrm{Ca}^{2+}$ exchange in cortical astrocytes. Glia 51:35-46

Gaetz M (2004) The neurophysiology of brain injury. Clin Neurophysiol 115:4-18

Gasser TC, Ogden RW, Holzapfel GA (2006) Hyperelastic modelling of arterial layers with distributed collagen fibre orientations. J R Soc Interface 3:15-35

Gennarelli TA, Spielman GM, Langfitt TW, Gildenberg PL, Harrington T, Jane JA, Marshall LF, Miller JD, Pitts LH (1982) Influence of the type of intracranial lesion on outcome from severe head injury. J Neurosurg 56:26-32

Gentleman SM, Roberts GW, Gennarelli TA, Maxwell WL, Adams JH, Kerr S, Graham DI (1995) Axonal injury: a universal consequence of fatal closed head injury. Acta Neuropathol 89:537-543

Hardy WN, Foster CD, Mason MJ, Yang KH, King AI, Tashman $S$ (2001) Investigation of head injury mechanisms using neutral density technology and high-speed biplanar x-ray. Stapp Car Crash J 45:337-368

Highley JR, Walker MA, McDonald B, Crow TJ, Esiri MM (2003) Size of hippocampal pyramidal neurons in schizophrenia. Br J Psychiatry $183: 414-417$

Ho J, von Holst H, Kleiven S (2009) An automatic method to generate a patient specific finite element head model. Int J Crashworthines 14:555-563

Hrapko M, van Dommelen JAW, Peters GWM, Wismans JSHM (2008) The influence of test conditions on characterization of the mechanical properties of brain tissue. J Biomech Eng 130:031003-1-10

Hutsler JJ (2003) The specialized structure of human language cortex: Pyramidal cell size asymmetries within auditory and language-associated regions of the temporal lobes. Brain Lang 86: $226-242$ 
Kleiven S (2007) Predictors for traumatic brain injuries evaluated through accident reconstruction. Stapp Car Crash J 51:81-114

Kouznetsova VG, Brekelmans WAM, Baaijens FPT (2001) An approach to micro-macro modeling of heterogenous materials. Comp Mech 27:37-48

Marieb EN (1998) Human anatomy and physiology, 4th edn. Benjamin/Cummings Science Publishing, Menlo Park

Marjoux D, Baumgartner D, Deck C, Willinger R (2008) Head injury prediction of the HIC, HIP, SIMon and ULP criteria. Accident Anal Prev 40:1135-1148

McCracken P, Manduca A, Felmlee J, Ehman R (2005) Mechanical transient-based magnetic resonance elastography. Magn Reson Med 53:628-639

McElhany JH, Roberts VL, Hilyard JF (1976) Handbook of human tolerance, 2nd edn. Japan Automobile Research Institute Inc., Tokyo

Miller RT, Margulies SS, Leoni M, Nonaka M, Chen X, Smith DH, Meaney DF (1998) Finite element modeling approaches for predicting injury in an experimental model of severe diffuse axonal injury. In: Stapp car crash conference proceedings, vol 42. The Stapp Association, pp 155-166

Morrison III B, Cater HL, Benham CD, Sundstrom LE (2006) An in vitro model of traumatic brain injury utilising two-dimensional stretch of organotypic hippocampal slice cultures. J Neurosci Methods 150:192-201

NHTSA (1972) Occupant crash protection-head injury criterion, S6.2 of FMVSS 571.208. NHTSA, Washington

Nicolle S, Lounis M, Willinger R (2004) Shear properties of brain tissue over a frequency range relevant for automotive impact situations: new experimental results. Stapp Car Crash J 48:239-258

Nicolle S, Lounis M, Willinger R, Palierne JF (2005) Shear linear behavior of brain tissue over a large frequency range. Biorheology 42:209-223

Ning X, Zhu Q, Lanir Y, Margulies SS (2006) A transversely isotropic viscoelastic constitutive equation for brainstem undergoing finite deformation. J Biomech Eng 128:925-933

Nolte J (2002) The human brain: an introduction to its functional anatomy, 5th edn. Mosby, Inc., St. Louis
Pierri JN, Volk CLE, Auh S, Sampson A, Lewis DA (2001) Decreased somal size of deep layer 3 pyramidal neurons in the prefrontal cortex of subjects with schizophrenia. Arch Gen Psychiatry 58:466473

Povlishock JT (1993) Pathobiology of traumatically induced axonal injury in animals and man. Ann Emerg Med 22:980-986

Prange MT, Margulies SS (2002) Regional, directional, and age-dependent properties of the brain undergoing large deformation. J Biomech Eng 124:244-252

Prange MT, Meaney DF, Margulies SS (2000) Defining brain mechanical properties: Effects of region, direction, and species. Stapp Car Crash J 44:205-213

Rajkowska G, Goldman-Rakic PS (1995) Cytoarchitectonic definition of prefrontal areas in the normal human cortex: I. Remapping of areas 9 and 46 using quantitative criteria. Cereb Cortex 5:307-322

Rajkowska G, Selemon LD, Goldman-Rakic PS (1998) Neuronal and glial somal size in the prefrontal cortex. Arch Gen Psychiatry $55: 215-224$

Shaw NA (2002) The neurophysiology of concussion. Prog Neurobiol 67:281-344

Smith DH, Meaney DF, Shull WH (2003) Diffuse axonal injury in head trauma. J Head Trauma Rehabil 18:307-316

Tagliaferri F, Compagnone C, Korsic M, Servadei F, Kraus J (2006) A systematic review of brain injury epidemiology in Europe. Acta Neurochir 148:255-268

Takhounts EG, Eppinger RH, Campbell JQ, Tannous RE, Power ED, Shook LS (2003) On the development of the SIMon finite element head model. Stapp Car Crash J 47:51-57

Viano DC, Casson IR, Pellman EJ, Zhang L, King AI, Yang KH (2005) Concussion in professional football: brain responses by finite element analysis: part 9. Neurosurgery 57:891-916

Wright RM, Ramesh KT (2012) An axonal strain injury criterion for traumatic brain injury. Biomech Model Mechanobiol 11:245-260. doi:10.1007/s10237-011-0307-1

Zhang L, Yang KH, King AI (2004) A proposed injury threshold for mild traumatic brain injury. J Biomech Eng 126:226-236 